\title{
Delays in Presentation in Patients With Acute Myocardial Infarction During the COVID-19 Pandemic
}

\author{
Ali Aldujeli ${ }^{\mathrm{a}, \mathrm{e}}$, Anas Hamadeh ${ }^{\mathrm{b}, \mathrm{c}}$, Kasparas Briedis ${ }^{\mathrm{a}}$, Kristen M. Tecson ${ }^{\mathrm{c}}$, Joshua Rutland ${ }^{\mathrm{b}, \mathrm{c}}$, \\ Zilvinas Krivickas ${ }^{a}$, Simas Stiklioraitis ${ }^{a}$, Kamilija Briede ${ }^{a}$, Montazar Aldujeilid, \\ Ramunas Unikas $^{\mathrm{a}}$, Diana Zaliaduonyte ${ }^{\mathrm{a}}$, Remigijus Zaliunas ${ }^{\mathrm{a}}$, Ravi C. Vallabhan ${ }^{\mathrm{b}, \mathrm{c}}$, \\ Peter A. McCullough ${ }^{\mathrm{b}, \mathrm{c}}$
}

\begin{abstract}
Background: The coronavirus disease 2019 (COVID-19) pandemic, caused by severe acute respiratory syndrome coronavirus 2 (SARSCoV-2) virus, has had a major impact on the behavior of patients, as well as on the delivery of healthcare services. With older and more medically vulnerable people tending to stay at home to avoid contracting the virus, it is unclear how the behavior of people with acute myocardial infarction (AMI) has changed. The aim of this study was to determine if delays in presentation and healthcare service delivery for AMI exist during the COVID-19 pandemic compared to the same period a year prior.
\end{abstract}

Methods: In this single-center, retrospective study, we evaluated patients admitted with ST-segment elevation myocardial infarction (STEMI) or non-ST-segment elevation myocardial infarction (NSTEMI) during early months of the COVID-19 pandemic (March 11, 2020 to April 20, 2020) compared to patients admitted with same diagnosis during the same period a year prior.

Results: There were 30 and 62 patients who presented with NSTE$\mathrm{MI}$ in the pandemic and pre-pandemic eras, respectively. The median pain-to-door time was significantly larger during the pandemic compared to pre-pandemic era $(1,885(880,5,732)$ vs. $606(388$, 944) $\min , \mathrm{P}<0.0001)$. There was a significant delay in door-toreperfusion time during the pandemic with a median time of 332 $(182,581)$ vs. $194(92,329) \min (\mathrm{P}=0.0371)$. There were $24(80 \%)$ and $25(42 \%)$ patients who presented after $12 \mathrm{~h}$ of pain onset in pandemic and pre-pandemic eras, respectively $(\mathrm{P}=0.0006)$. There were 47 and 60 patients who presented with STEMI during the pandemic timeframe of study and pre-pandemic timeframe, respectively. The

Manuscript submitted September 30, 2020, accepted October 22, 2020

Published online November 2, 2020

${ }^{a}$ Lithuanian University of Health Sciences, Kaunas, Lithuania

bBaylor University Medical Center, Dallas, TX, USA

'Baylor Heart and Vascular Institute, Dallas, TX, USA

dUniversity of Brescia, Brescia, Italy

${ }^{e}$ Corresponding Author: Ali Aldujeli, Lithuanian University of Health Sciences, Eiveniu Street 2, Kaunas 50161, Lithuania.

Email: Ali.Aldujeli@kaunoklinikos.lt

doi: https://doi.org/10.14740/cr1175 median pain-to-door time during the pandemic was significantly larger than that of the pre-pandemic $(620(255,1,500)$ vs. $349(146$, $659)$ min, $\mathrm{P}=0.0141)$. There were $22(47 \%)$ and $14(24 \%)$ patients who presented after $12 \mathrm{~h}$ of pain onset in the pandemic and prepandemic eras, respectively $(\mathrm{P}=0.0127)$. There was not a significant delay in door-to-reperfusion time $(\mathrm{P}=0.9833)$. There were no differences in in-hospital death, stroke, or length of hospitalization between early and late presenters, as well as between pandemic and pre-pandemic eras.

Conclusions: In conclusion, this study found that patients waited significantly longer during the pandemic to seek medical treatment for AMI compared to before the pandemic, and that pandemic-specific protocols may delay revascularization for NSTEMI patients. These findings resulted in more than a threefold increase from the onset of symptoms to revascularization increasing the risks for future complications such as left ventricular dysfunction and cardiovascular death. Efforts should be made to increase patients' awareness regarding consequences of delayed presentation, and to find a balance between hospital evaluation strategies and goals of minimizing total ischemic time.

Keywords: COVID-19; SARS-CoV-2; Delayed revascularization; COVID-19 fear; Acute myocardial infarction

\section{Introduction}

The World Health Organization declared coronavirus disease 2019 (COVID-19) a pandemic on March 11, 2020 [1]. Since then, concerns have grown that patients with heart disease are avoiding care due to fear of contracting COVID-19. However, this phenomenon is not clearly understood. Reports from northern Italy revealed a significant decrease in acute coronary syndrome hospitalizations during the early days of the COVID-19 pandemic [2]. Similarly, investigators in Hong Kong described significant delays in a small number of patients with ST-segment elevation myocardial infarction (STEMI) seeking care after instituting local infection control measures [3]. Understanding the barriers to seeking and providing cardiovascular care is critical during these times. Hence, this study aimed to identify potential delays in seeking medical care for patients 
with acute myocardial infarction (AMI), as well as delays in revascularization for patients with non-ST-segment elevation myocardial infarction (NSTEMI) and STEMI.

\section{Materials and Methods}

Consecutive patients from March 11, 2020 to April 20, 2020 who presented with AMI and underwent invasive angiography at the Hospital of the Lithuanian University of Health Sciences Kaunas Clinics were compared to patients admitted with same diagnosis during the same period in the year 2019. Patients included in this study were identified by ICD-10 codes for NSTEMI and STEMI. Data collected included patient demographics, comorbidities, cardiac catheterization procedural characteristics, echocardiography results, clinical course (ischemic or hemorrhagic strokes, cardiopulmonary resuscitation and hypotensive shock) and outcomes (length of stay and inpatient death). All patients were followed to the first event: hospital discharge or death.

Pain-to-door time was defined as the time from symptom onset to first hospital medical contact, excluding prehospital hospital contact. We dichotomized presentation times into early and late groups such that early presenters reported the onset of symptoms as $12 \mathrm{~h}$ or less and late presenters reported the onset of symptoms as more than $12 \mathrm{~h}$. Door-to-reperfusion time was defined as the time of arrival to the first passage of an intracoronary device. STEMI and NSTEMI were defined according to fourth universal definition of myocardial infarction [4]. Cardiogenic shock was defined as prolonged hypotension (systolic blood pressure $<90 \mathrm{~mm} \mathrm{Hg}$ ) with evidence of decreased organ perfusion caused by severe right or left ventricular dysfunction thought to be attributed to myocardial infarction or mechanical complications resulting from myocardial infarction. Left ventricular ejection fraction was assessed after revascularization and prior to hospital discharge. Successful percutaneous coronary intervention (PCI) was defined as the reduction of coronary artery lesion stenosis to $<$ $30 \%$. This study was approved by the Institutional Review Board of the Lithuanian University of Health Sciences. This study was conducted in compliance with all the applicable institutional ethical guidelines for the care, welfare and use of animals.

Continuous variables were skewed and are presented as median (25th percentile, 75th percentile). Categorical variables are presented as counts with proportions. Analyses were conducted to compare eras (pandemic vs. pre-pandemic) and were stratified based on presentation (NSTEMI or STEMI) in order to reduce extraneous variability. Differences in patient characteristics and outcomes between study years were assessed via Wilcoxon rank sum and Chi-square tests, or Fisher's exact test, as appropriate. Statistical analyses were performed using SAS version 9.4 (Cary, NC).

\section{Results}

There were 30 and 62 patients who presented with NSTEMI during the pandemic and pre-pandemic timeframes of study (Table 1). Most of these patients were men, with hypertension and obesity being the most common risk factors. All patients (during the pandemic timeframe) tested negative for COVID-19 by nasopharyngeal real-time reverse transcription polymerase chain reaction during hospitalization. The median pain-to-door time was significantly longer for the pandemic vs. pre-pandemic eras $(1,885(880,5,732)$ vs. $606(388,944) \mathrm{min}$, $\mathrm{P}<0.0001)$. There were $24(80 \%)$ and $25(42 \%)$ late presenters in the pandemic and pre-pandemic eras, respectively $(\mathrm{P}=$ 0.0006). There was a significant delay in door-to-reperfusion during the pandemic timeframe $(332(182,581)$ vs. 194 (92, 329) $\min , \mathrm{P}=0.0371$; Fig. 1). PCI as a revascularization strategy was performed more often during the pandemic compared to the prior year $(83 \%$ vs. $61 \%)$, and a higher rate of patients $(21 \%)$ underwent bypass surgery in the period prior to the pandemic compared to $6.7 \%$ during the pandemic. Post revascularization left ventricular ejection fraction was similar in both groups ( $43 \%$ vs. $48 \%, \mathrm{P}=0.1235)$. The median length of stay was similar for both timeframes $(6(5,9)$ vs. $5(4,7)$ days, $\mathrm{P}$ $=0.3138)$. In-hospital mortality was similar as well $(6.7 \%$ vs. $4.8 \%, \mathrm{P}=0.6597)$. Other procedural characteristics and outcomes are in Tables 1 and 2 .

There were 47 and 60 patients who presented with STEMI during the pandemic and pre-pandemic timeframes, respectively (Table 1). Patients were mostly men. Hypertension was the most common risk factor, followed by obesity in both timeframes. All patients (during the pandemic timeframe) tested negative for COVID-19 by nasopharyngeal real-time reverse transcription polymerase chain reaction during hospitalization. The median pain-to-door time during the pandemic was significantly larger compared to the year prior (620 (255, $1,500)$ vs. $349(146,659) \mathrm{min}, \mathrm{P}=0.0141)$; there were 22 $(47 \%)$ and $14(24 \%)$ late presenters in the pandemic and prepandemic eras, respectively $(\mathrm{P}=0.0127)$. There were no differences in door-to-reperfusion times during the study timeframes (Fig. 2). As shown in Table 1, the majority of patients underwent angiograms through right radial access. PCI was the most common strategy for revascularization. Post revascularization left ventricular ejection fraction was similar in both groups ( $43 \%$ vs. $40 \%, \mathrm{P}=0.44)$. The median length of stay was similar during the pandemic and pre-pandemic eras $(6(5,8)$ vs. $7(5,8.5)$ days, $\mathrm{P}=0.3222)$. Inpatient mortality rates did not differ between study timeframes (4.3\% vs. $6.7 \%$, $\mathrm{P}=0.6955)$. No significant differences were detected for oral medications received on discharge between the two groups. Additional procedural characteristics and outcomes are in Tables 1 and 2 .

\section{Discussion}

In this study of nearly 200 AMI patients, we found evidence of significant delays in hospital presentation for patients under study during the pandemic compared to those during the same 5 weeks of the year before the pandemic. In particular, not only did NSTEMI patients wait nearly three times as long during the pandemic to seek care compared to their 


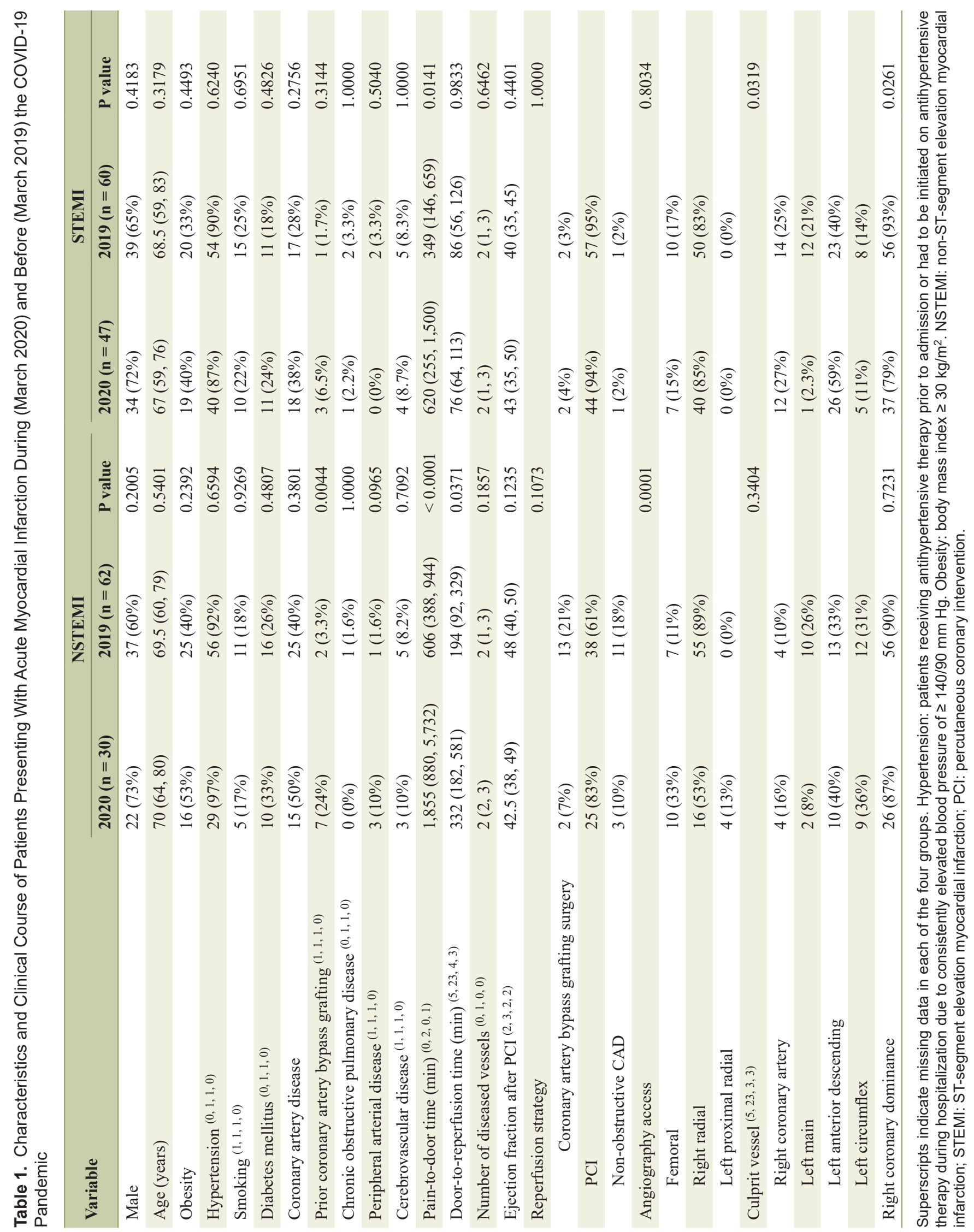




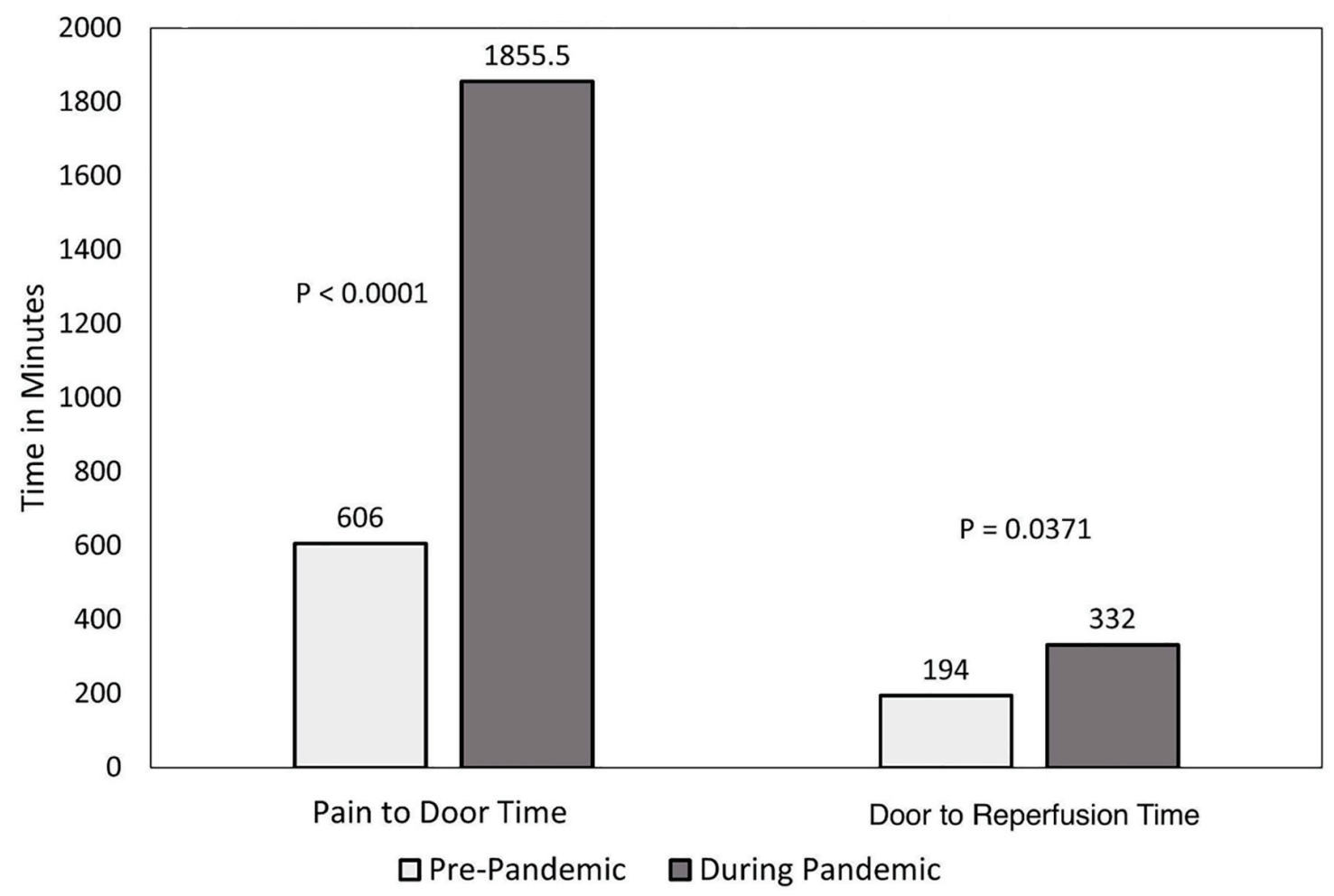

Figure 1. Time delays in patients presenting with NSTEMI. NSTEMI: non-ST-segment elevation myocardial infarction.

pre-pandemic counterparts, but they also experience significantly longer wait times to revascularization once hospitalized. STEMI patients waited nearly twice as long to present, but did not have longer times to revascularization once in the hospital. Medications on discharge were prescribed according European Society of Cardiology (ESC) recommendations for management of STEMI and NSTEMI $[5,6]$. Both the rates of left ventricular dysfunction and mortality were numerically higher, but these differences did not meet statistical significance in our small sample.

Longer time-to-presentation in patients with AMI is associated with higher rates of mortality $[7,8]$. In fact, the risk of 1-year mortality reportedly increases by $7.5 \%$ for every 30 min delay in treatment for patients presenting with STEMI [9].
Animal models have demonstrated that infarct size is significantly associated with duration of coronary artery occlusion [10]. Although we observed significantly longer pain-to-door times for patients with AMI during the pandemic, we did not detect a difference in these patients' ejection fraction, length of hospitalization, or rates of inpatient death.

Hospital systems throughout the world have had to implement pandemic-specific protocols in attempts to increase the safety of patients and providers alike by containing the spread of the virus [11]. However, our results indicate that these measures may have an impact on time-to-revascularization for patients presenting with NSTEMI. In general, a patient who presents with a STEMI would still be taken immediately to the catheterization lab for emergent revascularization with-

Table 2. Outcomes of Patients Presenting With Acute Myocardial Infarction During (March 2020) and Before (March 2019) the COVID-19 Pandemic

\begin{tabular}{|c|c|c|c|c|c|c|}
\hline \multirow{2}{*}{ Outcome } & \multicolumn{3}{|c|}{ NSTEMI } & \multicolumn{3}{|c|}{ STEMI } \\
\hline & $2020(n=30)$ & $2019(n=62)$ & P value & $2020(n=47)$ & $2019(n=60)$ & P value \\
\hline In-hospital hemorrhagic stroke $(1,0,0,1)$ & $0(0 \%)$ & $0(0 \%)$ & - & $0(0 \%)$ & $0(0 \%)$ & - \\
\hline In-hospital cardiopulmonary resuscitation & $2(6.7 \%)$ & $3(4.8 \%)$ & 0.6597 & $2(4.3 \%)$ & $4(6.7 \%)$ & 0.6930 \\
\hline Hypotension requiring vasopressors & $4(13 \%)$ & $5(8.1 \%)$ & 0.4665 & $5(10.6 \%)$ & $6(10 \%)$ & 1.0000 \\
\hline
\end{tabular}

Superscripts indicate missing data in each of the four groups. STEMI: ST-segment elevation myocardial infarction; NSTEMI: non-ST-segment elevation myocardial infarction. 


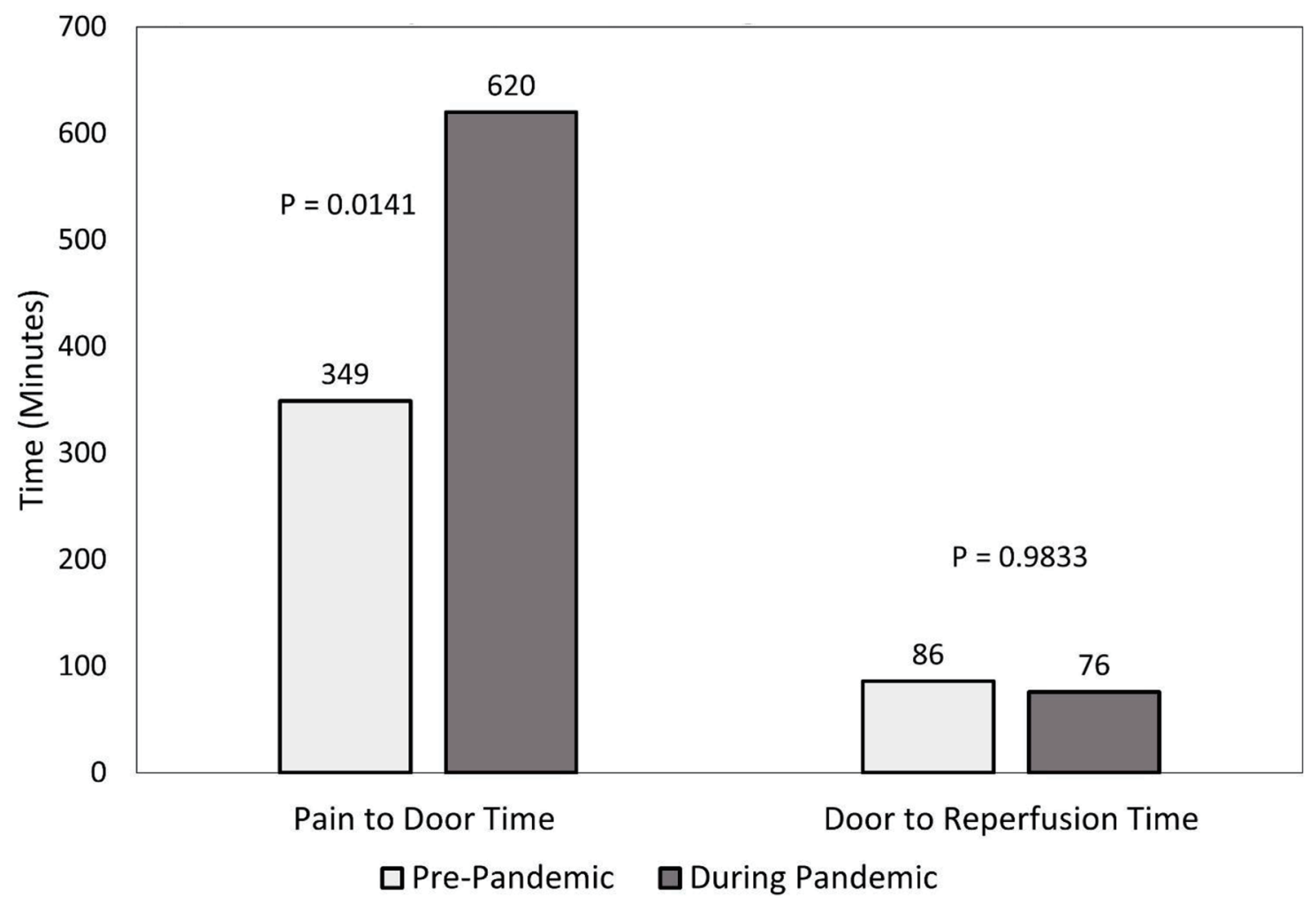

Figure 2. Time delays in patients presenting with STEMI. STEMI: ST-segment elevation myocardial infarction.

out waiting for COVID-19 testing, provided that the patient was not exhibiting symptoms of the infection. However, a patient presenting with an NSTEMI would be more likely to undergo COVID-19 testing prior to receiving invasive revascularization; we suspect this explains the door-to-reperfusion delays in our study.

In select cities in the United States and England, emergency department (ED) visits declined by nearly 50\% since shelter-in-place orders were activated $[12,13]$. Further, France observed a transient doubling of out-of-hospital cardiac arrests, as well as reduced survival rate during the pandemic [11]. In our small study alone, we observed a 37\% decrease in AMI hospitalizations. We suspect the reduction in crude AMI hospitalization events may be attributed to patients' fear of contracting COVID-19 infection at the ED and/or physicians' offices. These observations are highly concerning from a cardiovascular health standpoint, as the occurrence of dead on arrival becomes an increasing reality for those delaying treatment. Every effort should be taken to increase awareness of the consequences in delaying treatment, perhaps through utilizing telemedicine and outreach programs.

\section{Limitations}

This study has all the limitations inherent to small retrospective studies. Follow-up for these patients was limited to death or discharge, which is likely too short to detect any difference in major outcomes. Similarly, the sample size was mod- est which limited our power to detect significant differences in the rates of left ventricular dysfunction or cardiovascular death. Moreover, the study did not include COVID-positive patients which could have different outcomes [14]. There were missing data points for some procedural characteristics and outcomes.

\section{Conclusions}

In conclusion, this study suggests that patients are postponing by a period of threefold the needed medical and interventional care for AMI, and that pandemic-specific protocols may further delay revascularization for NSTEMI patients. Therefore, all efforts should be made to increase patients' awareness, as well as to adjust hospital evaluation strategies to minimize total ischemic time.

\section{Acknowledgments}

None to declare.

\section{Financial Disclosure}

This work was funded, in part, by the Baylor Health Care System Foundation, and by the National Association of Interventional Cardiology. 


\section{Conflict of Interest}

None to declare.

\section{Informed Consent}

Patients were not required to give informed consent to the study because the analysis used anonymous clinical data that were obtained after each patient agreed to treatment by written consent.

\section{Author Contributions}

Ali Aldujeli, MD, MSc: supervision, conceptualization, data curation, methodology, writing - original draft preparation, writing - reviewing and editing. Anas Hamadeh, MD: supervision, conceptualization, investigation, validation, writing reviewing and editing. Kasparas Briedis MD, MSc: conceptualization, investigation - data collection, validation, writing - reviewing and editing. Kristen $\mathrm{M}$. Tecson, $\mathrm{PhD}$ : formal analysis, writing - reviewing and editing, visualization, funding acquisition. Joshua Rutland, MD, MSc: writing - reviewing and editing. Zilvinas Krivickas: investigation - data collection, validation, writing - reviewing and editing. Montazar Al Dujeili, MD: writing - reviewing and editing. Simas Stiklioraitis: investigation - data collection, validation, writing - reviewing and editing. Kamilija Briede, MD: investigation - data collection, validation, writing - reviewing and editing. Ramunas Unikas, MD, MSc, PhD: writing - reviewing and editing. Diana Zaliaduonyte, MD, MSc, PhD: writing - reviewing and editing. Remigijus Zaliunas, MD, MSc, PhD: writing - reviewing and editing. Ravi C. Vallabhan, MD: writing - reviewing and editing. Peter A. McCullough, MD, MPH: writing - reviewing and editing.

\section{Data Availability}

The data supporting the findings of this study are available from the corresponding author upon reasonable request.

\section{References}

1. Mahase E. Covid-19: WHO declares pandemic because of "alarming levels" of spread, severity, and inaction. BMJ. 2020;368:m1036.

2. De Filippo O, D'Ascenzo F, Angelini F, Bocchino PP, Conrotto F, Saglietto A, Secco GG, et al. Reduced Rate of Hospital Admissions for ACS during COVID-19 outbreak in Northern Italy. N Engl J Med. 2020;383(1):88-89.

3. Tam CF, Cheung KS, Lam S, Wong A, Yung A, Sze M, Lam YM, et al. Impact of coronavirus disease 2019
(COVID-19) outbreak on ST-segment-elevation myocardial infarction care in Hong Kong, China. Circ Cardiovasc Qual Outcomes. 2020;13(4):e006631.

4. Thygesen K, Alpert JS, Jaffe AS, Chaitman BR, Bax JJ, Morrow DA, White HD, et al. Fourth Universal Definition of Myocardial Infarction (2018). J Am Coll Cardiol. 2018;72(18):2231-2264.

5. Ibanez B, James S, Agewall S, Antunes MJ, BucciarelliDucci C, Bueno H, Caforio ALP, et al. 2017 ESC Guidelines for the management of acute myocardial infarction in patients presenting with ST-segment elevation: The Task Force for the management of acute myocardial infarction in patients presenting with ST-segment elevation of the European Society of Cardiology (ESC). Eur Heart J. 2018;39(2):119-177.

6. Roffi M, Patrono C, Collet JP, Mueller C, Valgimigli M, Andreotti F, Bax JJ, et al. 2015 ESC guidelines for the management of acute coronary syndromes in patients presenting without persistent ST-segment elevation. Rev Esp Cardiol (Engl Ed). 2015;68(12):1125.

7. Bugiardini R, Ricci B, Cenko E, Vasiljevic Z, Kedev S, Davidovic G, Zdravkovic M, et al. Delayed care and mortality among women and men with myocardial infarction. J Am Heart Assoc. 2017;6(8):e005968.

8. Mol KA, Rahel BM, Meeder JG, van Casteren BC, Doevendans PA, Cramer MJ. Delays in the treatment of patients with acute coronary syndrome: Focus on pre-hospital delays and non-ST-elevated myocardial infarction. Int J Cardiol. 2016;221:1061-1066.

9. De Luca G, Suryapranata H, Ottervanger JP, Antman EM. Time delay to treatment and mortality in primary angioplasty for acute myocardial infarction: every minute of delay counts. Circulation. 2004;109(10):1223-1225.

10. Garcia-Dorado D, Theroux P, Elizaga J, Galinanes M, Solares J, Riesgo M, Gomez MJ, et al. Myocardial reperfusion in the pig heart model: infarct size and duration of coronary occlusion. Cardiovasc Res. 1987;21(7):537544.

11. Briedis K, Aldujeli A, Aldujeili M, Briede K, Zaliunas R, Hamadeh A, Stoler RC, et al. Considerations for management of acute coronary syndromes during the SARS-CoV-2 (COVID-19) pandemic. Am J Cardiol. 2020;131:115-119.

12. Wong LE, Hawkins JE, Langness S. Where are all the patients? Addressing COVID-19 fear to encourage sick patients to seek emergency care. NEJM Catalyst Innovations in Care Delivery. 2020;1:3.

13. Public Health England EDSSS. England. Year 2020, week 13. 1 April 2002. https://assets.publishing.service. gov.uk/government/uploads/system/uploads/attachment_ data/file/877600/EDSSSBulletin2020wk13.pdf.pdf.

14. Hamadeh A, Aldujeli A, Briedis K, Tecson KM, SanzSanchez J, Al Dujeili M, Al-Obeidi A, et al. Characteristics and outcomes in patients presenting with COVID-19 and ST-segment elevation myocardial infarction. Am J Cardiol. 2020;131:1-6. 